REGARDS

SUR L'ECONOMIE ALLEMANDE

BULLETIN ECONOMIQUE DU CIRAC
Regards sur l'économie allemande

Bulletin économique du CIRAC

98-99| 2010

Varia

\title{
Les médias dans l'Allemagne unie. De l'unification démocratique à la normalisation du marché
}

Isabelle Bourgeois

\section{(2) OpenEdition}

Édition électronique

URL : http://journals.openedition.org/rea/4191

DOI : $10.4000 /$ rea. 4191

ISBN : 978-2-8218-0892-8

ISSN : 1965-0787

\section{Éditeur}

CIRAC

\section{Édition imprimée}

Date de publication : 26 octobre 2010

Pagination : 63-78

ISSN : 1156-8992

\section{Référence électronique}

Isabelle Bourgeois, « Les médias dans l'Allemagne unie. De l'unification démocratique à la normalisation du marché », Regards sur l'économie allemande [En ligne], 98-99 | octobre 2010, mis en ligne le 01 octobre 2012, consulté le 14 novembre 2019. URL : http://journals.openedition.org/rea/ 4191 ; DOl : 10.4000/rea.4191 


\title{
Les médias dans l'Allemagne unie. De l'unification démocratique à la normalisation du marché
}

\author{
Isabelle Bourgeois
}

\begin{abstract}
20 ans après l'Unité, l'Allemagne est sans conteste le marché médiatique de loin le plus dynamique d'Europe. Mais alors que, dans les autres branches ou secteurs de l'économie, le processus d'unification s'effectuait somme toute sans difficultés majeures puisque les lois présidant à l'activité ne diffèrent que peu dans le monde, et qu'il suffit au fond de servir intelligemment l'offre et la demande, l'Unité médiatique de l'Allemagne avait tout d'une gageure. Car c'est dans le 'secteur' des médias que s'exprimait dans sa version la plus pure la partition du monde en deux systèmes politiques et idéologiques antagonistes: en une démocratie et une dictature. Les deux Allemagne étaient en guerre, du moins par ondes interposées, seules armes franchissant le Mur en 1989.
\end{abstract}

La libération du contenu - de l'information, des programmes - était une chose. La démocratisation de la presse s'est rapidement accomplie en RDA durant la "révolution pacifique ", aussi sous la pression d'un besoin de libre expression trop longtemps muselé. Mais de là à concevoir pour l'audiovisuel public des structures institutionnelles correspondant aux missions démocratiques des médias, et à les rendre de surcroît pérennes, il y avait un monde. Bien que prévue dans le Traité d'unification, l'unité des radios et TV publiques mit longtemps à se faire.

Certes, le modèle ouest-allemand, qui avait fait ses preuves (voir dans ce numéro la contribution d'U. Kammann), s'imposera à l'est aussi, devenant celui de toute l'Allemagne. Mais à l'époque de la chute du Mur, ce modèle subissait luimême de profondes mutations. Au plan réglementaire, le marché audiovisuel venait de s'ouvrir à des opérateurs privés, ce qui créait une situation de concurrence nouvelle. Au plan technique, on voyait se multiplier les vecteurs avec l'extension du réseau câblé et la réorganisation des fréquences hertziennes qui permettaient progressivement aux jeunes chaînes privées de se développer. Mais c'est à la veille de la chute du Mur, à la fin août 1989, qu'allait se déclencher une révolution dont on n'a pas, à l'époque, réellement entrevu la portée, tant les esprits furent accaparés au même moment par les prémisses de la chute du Mur: le lancement du premier satellite Astra. L'extension brutale du marché (ouest) que déclencha la chute du Mur, conjuguée à la démultiplication soudaine des vecteurs (satellite), a créé un formidable appel d'air, brouillant toutes les positions établies. Et c'est dans ce contexte en plein mouvement que se posait la question de l'unification de structures publiques de l'audiovisuel longtemps ennemies. Autant dire que les négociations furent mouvementées...

\section{RDA : agitation et propagande}

A la fin 1988, le gouvernement de la RDA interdit la vente du magazine satirique soviétique pour les jeunes : Sputnik. C'est probablement là la goutte d'eau qui fait déborder le vase, ou du moins un de ces événements qui, avec les élections truquées de la même année, préfigurent la fin immimente de l'emprise du régime de la RDA sur l'opinion. A la fin des années 1980, le parti-Etat SED affronte en effet une crise majeure de politique intérieure : le processus de démocratisation engagé par la Pologne en 1980 et d'autres Etats de l'Est n'est certes relaté - quand il

Fin des années 1980 : la RDA se ferme à la glasnost 
Mais l'information TV franchit le Mur

l'est - qu'avec la plus parcimonieuse prudence, mais il n'en risque pas moins de 'contaminer' l'opinion de la RDA et d'inciter les travailleurs est-allemands à revendiquer eux aussi plus de droits. La politique de perestroïka (transformation) et de glasnost (transparence) que mène Michaïl Gorbatchev en Union soviétique menace de déstabiliser les fondements mêmes du régime de la RDA, puisqu'elle s'accompagne d'un débat sur le passé stalinien du PC soviétique et du PC allemand. Or c'est ce 'travail de mémoire' que risquait d'importer en RDA l'édition incriminée de Sputnik (littéralement: compagnon de route, satellite), avec pour corollaire la revendication de plus de droits démocratiques.

«Il a été mis obstacle à toutes les publications constructives sur les processus de transformation en Union soviétique, de même qu'à la reproduction de documents afférents publiés dans les quotidiens soviétiques », peut-on lire ainsi dans le Compte rendu du Parti, distribué lors du congrès de dissolution du parti SED le 8 décembre 1989 (ce texte rédigé, après la démission du Comité central cinq jours auparavant, par une poignée d'ex-dirigeants du parti, dont Egon Krenz et Günter Schabowski, a été rejeté par le Comité directeur; texte publié in : Schabowski, 1990). Le gouvernement cherche donc certes à limiter l'influence de ces débats par toute une série d'interdictions (concernant notamment des films), mais il ne peut plus éluder la question : il lui faut choisir ou bien de poursuivre, seul de tous les Etats du Bloc Est en pleine réforme, sa politique marxiste-léniniste en matière d'information et de propagande, ou bien l'infléchir vers plus de glasnost.

Pendant quelques semaines, il continuera à contrôler officiellement l'information, tout en menant une politique du laisser-faire face à une situation dont il est contraint d'accepter qu'il ne la contrôle plus en réalité. Car la RDA se trouve dans une situation unique parmi tous les Etats de l'Est: elle partage la même langue que l'Allemagne 'impérialiste' de l'autre côté du Mur, et les médias audiovisuels de la République fédérale apportent aux citoyens de la RDA une information libre et factuelle sur leur propre pays, relatant justement tous les faits et événements que la propagande officielle s'efforce de ne pas divulguer. Autrement dit : structurellement, l'impact de la propagande est sapé par un pluralisme de fait qui permet aux citoyens de comparer les informations reçues et d'en confronter les sources (Kammann, 2010 ; Bourgeois, 2009).

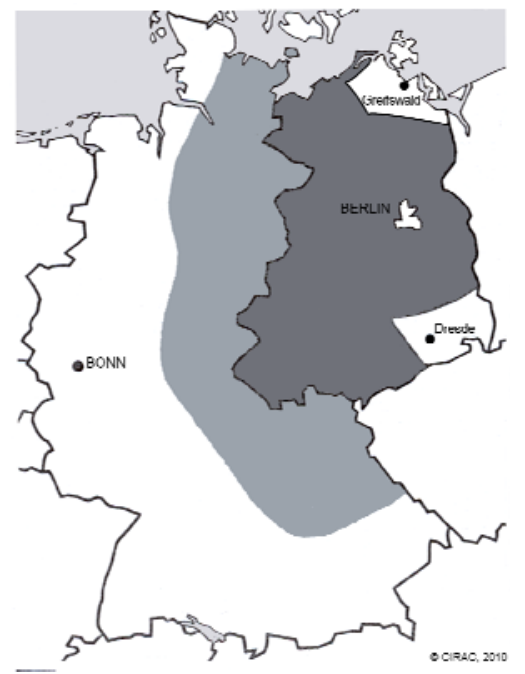

Les ondes ne s'arrêtent pas aux frontières. Le régime de la RDA s'était rendu à l'évidence dans les années 1970 en levant les sanctions à l'encontre des individus ayant dirigé leur antenne râteau vers l'Ouest, puis en lançant, à partir de cette période de « démarcation » (Abgrenzung) en réaction à l'Ostpolitik de la RFA, une 'contre-programmation' dans l'audiovisuel se traduisant principalement par l'adaptation idéologiquement conforme de concepts d'émissions occidentaux. A l'Ouest, pendant ce temps, les télévisions publiques multipliaient les relais tout le long du Mur. Et à la fin des années 1980, tout le territoire de la RDA était couvert par les chaînes de la RFA (et la radio $D L F$ ) - sauf deux régions à l'extrême nord-est (les environs de Greifswald, près de la Baltique) et à l'extrême sud-est : la zone de concentration d'activités autour de Dresde, ce qui avait valu à cette dernière zone d'ombre le sobriquet de « Vallée des Innocents » (Tal der Ahnungslosen) et à la première chaîne publique de l'Ouest (diffusée par l'ARD) celui de " hors agglomération de Dresde » (ARD = Außer Raum Dresden). Mais depuis le début des années 1980, antennes collectives (câble) et paraboles n'étaient plus interdites ...

NB : Les chaînes de la RDA couvraient une partie du territoire de la RFA ; mais elles n'étaient guère regardées par les Allemands de l'Ouest, sauf à la fin 1989.

Au moment où la RDA fête son $40^{\circ}$ anniversaire et où, durant les célébrations, se déroule à Berlin (Est), le 7 octobre 1989, la première manifestation spontanée de l'histoire de la RDA après la répression sanglante de celle du 17 juin 1953 (depuis, toute manifestation spontanée était interdite), les médias de la RDA sont totalement aux ordres du régime. Certes, la Constitution de la RDA (art. 27, al. 1) stipulait que «Tout citoyen de la République démocratique allemande a le droit d'exprimer librement et publiquement son opinion... », mais la phrase se 
poursuivait ainsi : «... dans la conformité aux principes de la présente Constitution ». Ces principes dont le résultat était de restreindre la liberté d'expression se résumaient à la souscription totale au rôle dirigeant du parti SED et à la doctrine du «centralisme démocratique ». L'ensemble de la chaîne de production de l'information était donc placée sous la tutelle idéologique du Parti dont la politique exprimait la voix des travailleurs. «La direction du parti avait le monopole de l'information comme de l'organisation du parti. La communication entre la direction du parti et l'organisation était à sens unique, se faisant du haut vers le bas... Un retour n'était pas souhaité. Les informations transmises de la base vers le haut étaient maquillées, la recherche scientifique sur l'opinion était considérée comme inutile et fut supprimée. Les organisations de la base étaient exclues de la formation de la volonté de la centrale. Leur fonction se résumait à mobiliser les masses pour une ligne dictée d'en haut » (Compte rendu du parti, in: Schabowski, 1990).

L'information devant être fidèle à la ligne du Parti dont elle était l'expression, la profession de journaliste - un «métier politique » - était réglementée : à l'issue d'une année de formation au sein d'une rédaction, le stagiaire devait déposer sa candidature au département de journalisme de l'Université Karl-Marx de Leipzig, que les Allemands de l'Est avaient baptisé « le monastère rouge » (das rote Kloster; voir l'ouvrage publié sous ce titre par B. Klump). Une fois reçu, il y effectuait quatre années d'études sanctionnées par un diplôme attestant de ses compétences professionnelles comme de sa sujétion au régime. En effet, explique le « Dictionnaire du journalisme socialiste » édité en 1981 par ce département : « Le journaliste socialiste est fonctionnaire du parti de la classe ouvrière.... II contribue à consolider le lien de confiance du peuple envers le Parti et l'Etat. L'ensemble de son activité est foncièrement déterminé par le programme et les résolutions du Parti marxiste-léniniste de la classe ouvrière ainsi que par la Constitution de l'Etat socialiste » (cité par Holzweissig, 1989).

Bien que cet état de fait soit officiellement nié (on renvoyait alors à la première partie de l'article de la Constitution sur la liberté d'expression), la censure s'appliquait sous ses deux formes : a posteriori et préalable. La première forme est celle qu'on connaît le mieux : tout journaliste contrevenant encourait un blâme et des représailles en cas de récidive. Cette réalité entretenait l'auto-censure : les journalistes y « étaient amenés par la contrainte. Ces 'ciseaux dans la tête' permettaient à Erich Honecker de se prévaloir, face aux journalistes et hommes politiques occidentaux, du fait qu'il n'y aurait pas de censure en RDA » (Compte rendu du parti, in : Schabowski, 1990). En RDA, le droit de la presse relevait du pénal. Plus significative encore est la censure préalable, puisque c'est à travers elle que s'exprimait la doctrine marxiste-léniniste. Ainsi, une fois par semaine, tous les rédacteurs en chef étaient réunis à Berlin pour prendre les directives ou mots d'ordre de la semaine. Ceux-ci concernaient à la fois les informations à passer sous silence, le contenu des informations à diffuser, et la manière de les traiter. Le «Manuel du journalisme de la RDA » explique pourquoi : "Pour transmettre au lecteur une vision correcte de la réalité objective et de sa cohérence, il est procédé par le Parti au choix des informations à rendre publiques, de leur emplacement, de la hiérarchisation des faits relatés, comme de leur formulation, ainsi que de la composition des titres » (cité par Holzweißig, 1989).

Longtemps, ces pratiques sont restées peu connues, les divulguer était risqué. L'opinion n'en prit conscience qu'avec la démocratisation des médias, rendue officielle par la proclamation de la liberté de la presse et la nomination, le 7 novembre 1989, de Günter Schabowski aux fonctions de Secrétaire du comité central du SED chargé de l'information et de la politique des médias (donc ministre de l'information). G. Schabowski y avait été nommé par Egon Krenz à la condition expresse de l'être en qualité de «liquidateur des structures jusque-là administrées », se rappelle-t-il, ajoutant : " cela voulait dire : fin de la mise sous tutelle de la presse ». Et il raconte qu'une de ses premières mesures fut de «convier les rédacteurs en chef pour les informer qu'à l'avenir ce genre de réunion n'avait plus
« Le journaliste socialiste est fonctionnaire du parti »

Censure a posteriori et censure préalable

Fin 1989 : proclamation de la liberté de la presse 
L'opinion prend connaissance des mots d'ordre auparavant dictés à la presse..

... par le Politbureau une presse totalement sous contrôle du Parti : ...

... édition, impression, distribution et abonnements

$1^{\text {er }}$ avril 1990 : fin des subventions. Les lecteurs se désabonnent... de sens et qu'ils devraient faire tous seuls ce qu'ils avaient à faire » (Schabowski, 1990).

Dans son édition du 19 janvier 1990, le quotidien CDU est-allemand Neue Zeit publie alors de larges extraits de ces directives, repris par le quotidien ouest-allemand F.A.Z. trois jours plus tard. Nous n'en citerons ici que deux. Alors que la pénurie de biens de consommation, notamment alimentaires (voir dans ce numéro l'analyse de K. Brenke) est manifeste en RDA et que filtrent via la télévision ouest-allemande les informations sur une pénurie bien plus dramatique encore en Roumanie ou en Pologne, le mot d'ordre du 10 août 1989 est : « Dans nos journaux, nous ne formulerons pas la moindre critique quant aux problèmes d'approvisionnement ». Alors que, après l'ouverture de la frontière entre la Hongrie et l'Autriche (le 2 mai 1989), l'exode des Allemands de l'Est vers la RFA commence à prendre des proportions dramatiques, le mot d'ordre du 27 juillet 1989 est: « Nous ne nous intéresserons pas à la problématique des départs de RDA ».

Ces directives étaient fixées par le Politbureau du SED. Leur mise en œuvre était confiée à un membre du Comité central qui avait sous sa direction le Département Propagande (chargé de fixer la ligne politique générale) et le Département Agitation chargé de la mettre en œuvre, donc de veiller à sa diffusion, et dont dépendait l'Office de presse du président du Conseil des ministres. C'est cet Office qui délivrait les mots d'ordre hebdomadaires. Le même avait sous sa responsabilité l'agence centrale d'information ADN et présidait deux Comités d'Etat assumant la tutelle, l'un de la radio, l'autre de la télévision. Pour le dire autrement: l'agence ADN, l'office de radio et l'office de télévision étaient le bras prolongé du Politbureau qui avait par ailleurs son organe (le quotidien Neues Deutschland) et contrôlait également via l'Office de presse les publications des organisations de masse (par exemple Junge Welt) comme les journaux des partis d'opposition autorisés (dont ce Neue Zeit de la CDU de l'Est).

\section{Renouveau de la presse, puis normalisation}

La presse écrite, abondante au demeurant avec 550 titres pour 1000 habitants (Statistiques officielles de la RDA), n'était pas seulement contrôlée via la formation des journalistes ou l'emprise du Parti sur son contenu. Elle l'était aussi au niveau de la production. Le papier était contingenté, et la principale quote-part attribuée aux organes du parti. Ainsi, sur les 39 quotidiens existants, tirant à plus de 9 millions d'exemplaires au total, la part du lion (6,6 millions) allait aux 15 édités par le SED (dont Neues Deutschland et Berliner Tageszeitung). Les quotidiens des organisations de masse ayant un tirage total de près de 2 millions, les 18 titres des partis d'opposition autorisés par le régime (ils étaient dénommés Blockparteien) devaient se contenter d'un tirage d'un peu plus de 800000 seulement au total. S'ajoute à cela que la quasi-totalité de la presse était imprimée par une holding dépendant du comité central ; quant à la distribution auprès des abonnés et des points de vente autorisés, elle était un monopole de la poste. Enfin, la politique des bas prix voulait que les journaux soient largement subventionnés (332 millions de marks est en 1989).

On comprend mieux, dès lors, pourquoi tant de titres de l'ex-RDA se sont effondrés ou ont fait faillite peu après la démocratisation de leur rédaction (Kammann, 2010) : la presse devait faire simultanément l'apprentissage de la liberté d'information et des lois du marché. Personne, à l'époque, ne pouvait imaginer qu'un quotidien puisse être une entreprise obéissant à des lois économiques propres, personne ne maîtrisait ni la production ni la distribution de ce produit qu'est un journal. Jusqu'au 15 mai 1990, la Deutsche Post (RDA) avait le monopole de la distribution, les éditeurs ne connaissaient donc même pas leurs abonnés, ni encore moins leur lectorat.

La libération de la presse s'est faite durant une brève période d'effervescence qui s'accompagne de toutes les manifestations non seulement d'une concurrence 
naissante, mais aussi de la découverte même de ce qu'elle implique, et qui se prolongera quelques années, puisque l'adhésion de la RDA à la RFA provoque une redistribution des cartes dans l'ensemble de l'Allemagne unie. La crise latente se manifeste au moment de l'abandon, le $1^{\text {er }}$ avril 1990 , de la politique de subventionnement de la presse. Du jour au lendemain, celle-ci découvre les coûts réels, et les titres triplent le prix de l'exemplaire, ce qui entraîne une vague de désabonnements sans pareille : selon une lettre du ministre est-allemand de la poste, publiée par le quotidien Berliner Zeitung, 3,6 millions d'abonnements auraient été résiliés en la seule journée du $1^{\text {er }}$ avril 1990 , tous titres confondus (CIRAC MEDIA, $\mathrm{n}^{\circ} 3 / 90$ ). Cette chute s'explique toutefois aussi par une normalisation des habitudes de consommation des médias des Allemands de l'Est: rassurés désormais sur la qualité comme la fiabilité de l'information, les lecteurs n'ont plus besoin comme avant de multiplier les abonnements pour confronter la présentation des faits et chercher à saisir, au détour d'une phrase ou entre les traits d'une caricature, l'élément invalidant la propagande officielle ou du moins apportant un éclairage un tantinet plus pluraliste. Et puis, maintenant qu'il n'y a plus de frontière ni d'interdits, ils peuvent librement acheter et découvrir les grands quotidiens (supra-régionaux) et les périodiques de l'ouest.

Enfin, les Allemands de l'Est ont d'autres priorités en matière de médias à l'approche de l'union monétaire : ils ont un immense besoin de rattrapage en biens de consommation. Et plus particulièrement en récepteurs de radio ou de TV pour enfin pouvoir capter en couleur cette télévision ouest-allemande qu'ils ne recevaient jusqu'ici qu'en noir et blanc. Au moment du passage à la couleur dans la deuxième moitié des années 1960, la RDA avait en effet opté pour le SECAM français pour contrer le PAL ouest-allemand ; ces deux standards étaient néanmoins compatibles en noir et blanc.

Dans ce contexte de bouleversements, et profitant d'une sorte de vide juridique sur un marché qui émergeait, les quatre grands éditeurs ouest-allemands de quotidiens et périodiques (Bauer, Burda, Gruner+Jahr et Springer) avaient entrepris immédiatement après l'ouverture de la RDA de monter un système de distribution pour mieux vendre à l'est leurs propres produits. Mais ils s'étaient fait rappeler à l'ordre par l'Office fédéral des Cartels (RFA) : à l'ouest, le secteur de la distribution est interdit aux éditeurs de presse pour emprêcher tout monopole d'opinion. Et en RDA, le gouvernement issu des élections libres du 18 mars 1990 adopte un décret sur la distribution de la presse, entré en vigueur le 16 mai, qui libéralise ce secteur d'activité, mais en conformité avec la législation de l'ouest. Restait la question de l'impression. 13 des 15 imprimeries que comptait la RDA appartiennent au Parti, qui ne les cède que progressivement et après force tergiversations. Les rares rotatives accessibles sont donc sursaturées, et de surcroît obsolètes, ce qui interdit notamment la couleur.

S'engagent alors de nombreuses coopérations entre quotidiens est et ouest. A l'origine, elles étaient donc avant tout d'ordre financier, technique et commercial, mais les professionnels de l'ouest apportaient aussi leur soutien en termes de formation. A la méconnaissance héritée du modèle économique de la presse s'ajoute l'évolution du contexte: le 'modèle' est-allemand d'une presse purement idéologique est maintenant obsolète, et la demande des lecteurs est-allemands allait, une fois leur soif de débats politiques assouvie, au format classique du quotidien généraliste, pluraliste et politiquement indépendant. Et ce quotidien se devait - à l'est comme à l'ouest - de servir aussi la demande d'informations locales ou régionales, puisque dans le mediamix allemand (des deux côtés de l'ancien Mur), c'est la télévision surtout qui livre l'information nationale et internationale. Or il y avait en RDA 218 éditions régionales des titres du SED (39), donc un potentiel à optimiser, d'autant que nombre de ces éditions s'étaient démocratisées, et que leur lectorat les avait fidèlement accompagnés dans cet effort. $\mathrm{Ce}$ sont donc majoritairement les éditeurs régionaux de l'ouest qui, forts de leur expérience (et des coopérations engagées avec leurs homologues de l'est), mais aussi désireux d'étendre leurs parts de marché, vont se poser candidats notam-
... et s'équipent en récepteurs radio et $\mathrm{TV}$

16 mai 1990 :

fin du monopole de distribution

Reprise des quotidiens régionaux est par les éditeurs ouest 
Aucun supra-régional

Un marché de la presse normalisé concentré et pourtant pluraliste est-allemand

ment au rachat des 10 grands quotidiens régionaux de l'ex-SED (tirage total de plus de 3 millions d'exemplaires) quand la Treuhandanstalt les mettra en vente au début 1991 selon une procédure d'appel d'offre respectant à la fois le mieux disant social et le droit de la concurrence ouest-allemand (un seul titre par candidat). A l'été 1992, les nouveaux contours du paysage est-allemand de la presse prennent forme : les trois quarts des journaux de la RDA ont survécu ; seuls ont disparu les organes du parti, les titres de la 'nouvelle gauche' et les titres à trop faible tirage ; quelques nouveaux titres sont nés, surtout dans la zone de l'ancien Mur. A une exception près, les 31 quotidiens qui subsistent (sur 39 à l'origine) appartiennent tous à des éditeurs de l'ouest. Leur tirage varie entre 315000 exemplaires (Leipziger Volkszeitung) et 560400 (Freie Presse, Chemnitz) - soit le niveau de l'ouest que seul dépasse le leader incontesté : WAZ (Essen ; 653 100).

Le rêve (ou la hantise) des repreneurs était de voir l'un de ces titres grandir au point de devenir un quotidien supra-régional comme le sont le F.A.Z. (Francfort), le Süddeutsche Zeitung (Munich) ou le Handelsblatt (Düsseldorf). Les grands quotidiens 'nationaux' allemands sont en effet tous d'abord des régionaux qui, simplement, sont distribués aussi dans l'ensemble de l'Allemagne et à l'international. Les médias allemands présentent en effet tous une structure aussi polycentrique que celle de l'économie ou des pouvoirs administratifs ou politiques. Pour contrer cette concurrence potentielle, l'un d'entre eux : Die Welt, déménage même de Bonn à Berlin. Mais aucun titre de l'est ne parvient à franchir le saut hors de sa région, même pas le Berliner Zeitung auquel on accordait pourtant les plus fortes chances après le déménagement de la capitale de Bonn à Berlin ; il est resté berlinois, métropolitain, sans plus. II faut dire aussi qu'en l'espace de 20 ans, les nouvelles technologies et les 'nouveaux médias' ont considérablement changé la donne.

Le paysage allemand de la presse est aujourd'hui normalisé. Et il présente un fort degré de concentration au niveau des éditeurs. Alors qu'en 1991, le nombre d'éditeurs de quotidiens s'élève à 410 (on en comptait 358 à l'ouest en 1989), il est tombé depuis à quelque 350. Dans le même temps, le nombre de rédactions autonomes, c'est-à-dire pouvant servir plusieurs titres (119 à l'ouest et 37 en RDA en 1989), s'est stabilisé jusqu'à 2008 autour de 135, et ce dès 1993, année de crise économique, après avoir connu un pic de 158 en 1991. Le nombre d'éditions (1 344 à l'ouest, 291 en RDA en 1989) a connu une évolution semblable : après un boom jusqu'en 1995 (plus de 1600 éditions), il s'est stabilisé aux alentours de 1500 . Autrement dit, bien que le marché soit fortement concentré au niveau des sociétés ou groupes de presse, l'offre de journaux se caractérise par un grand pluralisme. Quant au tirage total (20,3 millions d'exemplaires à l'ouest en 1989), il n'est en 2008 que de 20,2 millions dans l'Allemagne unie, après avoir connu une baisse régulière depuis le boom de l'Unité enregistré en 1991 (27,3 millions). C'est là l'effet des mutations observées dans tous les pays industrialisés depuis la montée en puissance des médias en ligne qui captent consommateurs de contenus et budgets publicitaires. D'autre part, c'est l'effet de l'Unité même : l'extension du marché qu'elle représentait pour les éditeurs de l'ouest a déclenché une phase de restructuration puis de concentration des éditeurs. Les mutations étaient d'autant plus profondes qu'elles avaient pour parallèle le repositionnement de la presse dans le segment de l'audiovisuel, lui aussi alors en pleine phase de restructuration.

\section{Audiovisuel : la quadrature du cercle a trouvé une réponse}

Diversification de la presse dans l'audiovisuel privé
Les éditeurs de presse sont en effet les acteurs de la première heure de la libéralisation de l'audiovisuel en RFA ouest (Bourgeois, 1995). Ils y sont engagés selon leur taille et leur 'métier'. Les éditeurs de la presse régionale préfèrent la radio, autre 'métier' construit sur le verbe, et média local ou régional par excellence en Allemagne. Cela est dû au fait que les Länder sont souverains en matière d'audiovisuel et que, les ondes longues ayant été retirées à l'Allemagne au sortir de la guerre, la bande FM est exploitée en RFA dès le début de 1949 ; or elle ne des- 
sert que de courtes distances. En outre, les fréquences sont limitées en nombre par une gestion du spectre que rend délicate la présence des transmissions militaires des forces alliées. Quant aux grands groupes que sont les éditeurs de périodiques, média associant le verbe et l'image, ils s'investissent dans la télévision qui est lui aussi un média à diffusion nationale, tout comme leurs titres.

Comme une 'loi' implicite veut qu'aucun capital étranger à la branche (sauf bancaire, bien sûr) ne puisse être investi dans les médias, la croissance rapide du secteur privé de l'audiovisuel après le feu vert que lui donna l'entrée en vigueur, le $1^{\text {er }}$ avril 1987, du Contrat d'Etat sur l'ordre dual de la radiodiffusion, a toujours été organique ou pour le dire autrement: endogame. Le même constat vaut a fortiori pour ses permanentes restructurations. Cette interdiction tacite - le 'tabou Hugenberg' - vise à interdire à tout jamais la collusion d'intérêts entre industrie (notamment militaire), monde politique et médias comme ce fut le cas sous le $\mathrm{III}^{\mathrm{e}}$ Reich. Sour la République de Weimar, Alfred Hugenberg, président du directoire de Krupp et député nationaliste, avait constitué un empire médiatique qui fut le levier de l'accession au pouvoir d'Hitler et favorisa la mise au pas des médias (surtout audiovisuels) dès l'entrée en fonctions du ministre de la Propagande, Josef Goebbels. En se constituant en 1949, la jeune RFA a ainsi doublement tiré les enseignements de la dictature nazie : par ce tabou concernant le capital des médias, et par le principe de la liberté constitutionnelle des médias. Pour assumer pleinement leurs fonctions d'information, d'articulation, de critique et de contrôle en démocratie, les médias doivent en effet être indépendants non seulement du pouvoir politique (exécutif, en l'occurrence), mais de tout pouvoir, notamment économique.
Des médias qui croissent par 'endogamie'

\section{La portée de la liberté de l'information en RFA}

La loi fondamentale garantit la liberté d'information. Cette garantie figure presque en tête du catalogue des droits fondamentaux. C'est l'art. 5, al.1: "Chacun a le droit d'exprimer et de diffuser librement son opinion par la parole, par l'écrit et par l'image, et de s'informer sans entraves aux sources qui sont accessibles à tous. La liberté de la presse et la liberté d'informer par la radio, la télévision et le cinéma sont garanties. Il n'y a pas de censure ». L'al. 2 poursuit: " ces droits trouvent leurs limites dans les prescriptions des lois générales, dans les dispositions légales sur la protection de la jeunessse et dans le droit au respect de l'honneur personnel ». (L'al. 3 garantit la liberté de l'art, de la science, de la recherche et de l'enseignement).

Dans son abondante jurisprudence, le Tribunal constitutionnel fédéral n'a cessé d'insuffler vie à cette liberté dont, dans son premier grand arrêt rendu en 1961 (surnommé « jugement sur la TV Adenauer » et considéré comme la 'Grande charte' de l'audiovisuel), il en a explicité la portée. "L'art. 5 de la Loi fondamentale contient plus que le simple droit fondamental individuel du citoyen au respect, de la part de l'Etat, d'un champ de liberté au sein duquel il peut exprimer librement son opinion. L'art. 5, alinéa 1, phrase 2 de la Loi fondamentale garantit en particulier l'autonomie de la presse en tant qu'institution... II serait contraire à cette garantie constitutionnelle que l'Etat, directement ou indirectement, réglemente ou dirige en totalité ou en partie la presse ». Autrement dit, outre-Rhin, la presse jouit d'une garantie institutionnelle de sa liberté.

Cette garantie institutionnelle s'applique également à l'audiovisuel (ou : radiodiffusion). Les juges de Karlsruhe poursuivent: « Abstraction faite de la spécificité de la radiodiffusion..., la radiodiffusion appartient, au même titre que la presse, aux moyens modernes de communication de masse indispensables qui influent sur l'opinion publique et contribuent à la former. La radiodiffusion est plus qu'un simple 'medium' dans la formation de l'opinion publique, elle en est bien plutôt un 'facteur' éminent ». II s'ensuit que, pour la radiodiffusion, «la liberté ne revêt pas une importance moindre que pour la presse. Cela est clairement exprimé dans l'art. 5 dont l'al. 1, phrase 2 garantit, en même temps que la liberté de la presse, 'la liberté de la relation par la radiodiffusion et le cinéma' ».

La différence entre presse et audiovisuel réside dans la forme que prend ou droit prendre le pluralisme. La presse étant un marché, soumis à vive concurrence, le pluralisme est de ce fait assuré - en externe, par la pluralité des produits et des positions exprimées. Une telle concurrence étant par nature limitée dans l'audiovisuel (du moins à l'ère analogique, et du moins en 1961) du fait de la rareté des fréquences, le pluralisme externe est difficile à réaliser. II convient donc de prévoir par la loi une structure organisationnelle permettant d'assurer ce pluralisme en interne.

« L'art. 5 exige... que cet instrument moderne de la formation de l'opinion ne soit livré ni à l'Etat, ni à un seul groupe social. Les organismes de radiodiffusion doivent donc être dotés d'une structure telle que l'ensemble des forces à prendre en considération disposent d'un pouvoir d'influence au sein des organes de la société et puissent s'exprimer dans le programme global », c'est-àdire dans l'ensemble des chaînes et radios produites en Allemagne, considéré comme un tout. Cette indépendance va très loin : même un législateur, pourtant élu du peuple, ne peut édicter la moindre directive en matière de programmes (arrêt de 1987).

Si les juges ont étendu le corollaire de ces garanties - le droit à l'information de tout un chacun - à l'ère du numérique en 2008 , aucun arrêt depuis 1961 n'a modifié leur approche. Alors qu'elle s'appliquait, à l'époque, au seul secteur public de l'audiovisuel, elle a simplement été étendue en 1986 au secteur privé naissant, introduisant dans le même temps un 'partage des rôles' entre public et privé dans la phase de constitution du marché : au service public d'assurer le " service de base » (missions d'intérêt général), aux jeunes chaînes privées d'assurer seulement des « standards de base ».

Le contrôle pluraliste par les groupes représentatifs de la société, qui s'effectue en interne dans le cas du public (via le Rundfunkrat, conseil de surveillance), est externalisé dans celui du privé, et confié à des autorités de régulation, indépendantes, régionales : les Landesmedienanstalten. 
Secteur public : comment marie l'eau et le feu?
Si, après la chute du Mur, les radios et TV privées de RFA cherchent à s'étendre à l'est, profitant elles aussi de cette extension du marché pour décupler leur audience et multiplier les 'contacts' publicitaires, et mues par l'espoir de conquérir quelques fréquences disponibles pour leur diffusion herztienne terrestre alors en plein développement, la situation était tout autre pour le secteur public. Le secteur privé était, quant au fond et à quelques détails près, aux prises avec les seules réalités du marché (et des plans de fréquences). Le secteur public, lui, était doté par définition d'une mission foncièrement politique: indépendant du pouvoir, « medium et facteur » de la démocratie d'un côté du Mur, instrument du "Parti marxiste-léniniste de la classe ouvrière » de l'autre. Le défi de l'Unité était alors de (ré)concilier structures, acteurs et programmes que tout opposait depuis 40 ans, en un mot: de marier l'eau et le feu.

\section{La 'guerre des ondes'}

Vitrines de la partition du monde, les deux Allemagne s'affrontaient bien sûr par ondes interposées.

- Au niveau de la radio, la radio internationale de la RDA : RBI (Radio Berlin international) diffusait vers l'Ouest la propagande officielle du SED. En face, les Alliés américains avaient installé à Munich la Voice of America, radio de contre-propagande ; et dans leur secteur à Berlin, le RIAS (Radio im amerikanischen Sektor), sorte de 'voix du monde libre'. La RFA pour sa part, dont la démocratie est établie sur la liberté d'information et dont la Constitution laissait ouverte la possibilité d'une adhésion de la RDA (c'est l'art. 23 qui permit l'Unité et qui fut modifié ensuite, ancrant la RFA dans l'Union européenne), préféra miser sur l'information. C'est ainsi que fut institué en 1960 une radio généraliste desservant l'Europe et 'l'autre Allemagne' : le Deutschlandfunk (Cologne). Elle émettait en ondes moyennes et sur la seule fréquence ondes longues restant à la RFA après la guerre ; celle-ci était tournée vers l'Est. Elle avait, en face, la radio 'européenne' de la RDA : Stimme der DDR.

- Au niveau de la télévision - rappelons que les programmes franchissaient le Mur - l'affrontement se faisait via les émissions. L'exemple le plus célèbre est ce magazine hebdomadaire de propagande (durée : 20 minutes) lancé en 1960 en RDA, en pleine guerre froide : «Der schwarze Kanal » (le « canal noir », noir comme l'impérialisme occidental). Il sera déprogrammé peu après la chute du Mur. Sa mission était de développer des stratégies 'de barrage' pour contrer l'impact de la TV de l'ouest; il utilisait pour ce faire des extraits d'émissions ou d'articles parus en RFA pour 'informer' sur les dysfonctionnements d'un monde capitaliste traversant crise après crise et mieux faire apparaître, par contraste, les mérites du régime socialiste. En réaction à la " politique de démarcation » de la RDA, la deuxième chaîne ouest-allemande (ZDF) lance en 1971 un magazine politique hebdomadaire d'une demi-heure qui se veut l'émission par excellence d'une Allemagne unie: «Kennzeichen D» ( Matricule D », D comme Deutschland). Sa mission est double : servir de correctif à la propagande de la RDA, mais aussi et avant tout à l'effet trompeur que pouvaient avoir sur des téléspectateurs est-allemands délibérément tenus à l'écart des réalités ouest-allemandes les programmes de la RFA (et la publicité). Réputée pour l'honnêteté et l'objectivité de son information, elle était très prisée des Allemands de l'Ouest et de l'Est. Pour ces derniers, elle était l'une des sources d'information les plus crédibles sur la RDA.

RDA : 6 radios... Au moment de la révolution pacifique, le Comité d'Etat pour la Radio dépendant du Département Agitation a sous sa tutelle l'Office de radio et ses 5 divisions éditant 6 stations : Radio DDR qui diffuse deux programmes (Radio DDR 1, informations 24 heures sur 24, et Radio DDR 2, émissions culturelles en après-midi et soirée) ; Berliner Rundfunk (radio métropolitaine, 24 heures sur 24) ; Stimme der DDR (radio 'européenne'), Radio Berlin International (la « voix » de la RDA à l'international) et Jugendradio DT 64 (pour les jeunes, créée en 1987). En matière d'information générale, les Allemands de l'Est préfèrent les radios de l'Ouest, principalement RIAS (à Berlin) et Deutschlandfunk qu'ils peuvent capter dans toute la RDA. Quant aux jeunes, ils sont attirés par la musique occidentale, ce qui avait incité le Parti à leur proposer DT 64 avec une 'contre-programmation' de rock ou folk idéologiquement plus conforme. II en ira de même pour la TV qui, à l'instigation de Margot Honecker, crée le $1^{\text {er }}$ septembre 1989 une nouvelle émission pour les jeunes avec la même mission : « Elf 99 ».

... et 2 chaînes de TV L'Office de télévision Fernsehen der DDR qui dépend du Comité d'Etat pour la télévision (Département Agitation) réalise deux chaînes nationales : DDR 1 (généraliste ; elle émet de 8 heures à minuit) et DDR 2 (à dominante culturelle, avec quelques émissions régionales; de 17 heures en moyenne jusqu'à minuit). Elles diffusent un journal télévisé de 30 minutes : «Aktuelle Kamera », programmé à 19 heures 30 sur DDR 1, puis réédité dans la soirée (vers 22 heures) sur DDR 1 et $D D R 2$; l'information présentée est passée au crible de la censure préalable du Parti. Les téléspectateurs est-allemands boudaient cette vitrine de la propagande officielle, lui préférant de loin les JT de l'ouest, surtout le « Tagesschau » 
de la première chaîne publique de l'ARD (Bourgeois, 2009). Or à l'automne 1989, «Aktuelle Kamera » est méconnaissable : dès le 11 octobre, il informe sur les manifestations qui se déroulent à Leipzig depuis quelques jours (et qu'on appellera ensuite les « manifestations du lundi »). Ce JT s'est démocratisé comme l'avait fait la presse avant lui, et son nouveau ton critique fait qu'il est maintenant assidument suivi à l'Est comme à l'Ouest (dans la semaine du 6 au 12 novembre, il a eu en moyenne 320000 téléspectateurs par jour en RFA, soit dix fois plus qu'avant). Cet engouement retombera peu après, quand les événements seront moins dramatiques.

Ce dispositif existait depuis 1968, une date à la portée très différente en RDA et dans le monde occidental : cette année-là, la RDA adopte une nouvelle Constitution et, entre autres mesures, le Parti resserre les liens avec les médias; le département de journalisme de l'Université de Karl-Marx à Leipzig sera créé en 1969. Pour mieux contrôler l'audiovisuel (et notamment la TV qui prenait alors son essor), l'ancien Office central de radiodiffusion dépendant du Département Agitation est scindé en deux. L'Office pour la TV était dirigé depuis par Heinrich Adameck, cadre du Parti. En décembre 1989, ce dernier est démis de ses fonctions, et son poste supprimé. A la place est créé celui de Generalintendant (intendant général) ; il sera occupé par le journaliste Hans Bentzien qui avait été 'mis au placard' en 1978 (voir la contribution d'U. Kammann dans ce numéro). C'est lui qui est chargé de mener la démocratisation de l'Office de TV, qui reprend alors le nom qu'il portait avant la politique de démarcation: Deutscher Fernsehfunk (DFF).

Mais alors que la démocratisation et la normalisation s'effectuent très rapidement dans la presse, et que ce processus est largement achevé avant même que soit scellée l'Unité politique, il faudra trois bonnes années à l'audiovisuel de la RDA pour accomplir ce processus. Le 13 février 1990, la Volkskammer (le parlement de la RDA) institue un "Conseil de contrôle des médias » : le Medienkontrollrat. II est composé de 23 représentants des partis, des commissions parlementaires, des Eglises, du gouvernement et des diverses formations réunies au sein du Runder Tisch (la «Table ronde » qui s'était constituée lors de la révolution pacifique et avait conseillé le gouvernement Modrow jusqu'aux élections libres à la Volkskammer le 18 mars 1990). Ce Medienkontrollrat est chargé de couper le cordon ombilical entre le gouvernement et les médias et de garantir leur indépendance en application de la Loi sur la garantie de la liberté d'opinion, d'information et des médias entrée en vigueur le même jour. Cette loi rompt avec les principes marxistes-léninistes tels qu'ils s'appliquaient à l'information selon la Constitution de la RDA. Son art. 1 se substitue à l'art. 27 de la Constitution de la RDA; il énonçait: "Chaque citoyen a le droit à la libre expression de son opinion. Ce droit comprend la liberté d'accéder, sans considération de frontière, aux informations et idées de toute sorte, par la parole, l'écrit, l'imprimé, l'œuvre d'art ou par tout autre moyen de son choix, comme il comprend la liberté de les recevoir et de les communiquer » (CIRAC MEDIA, $\left.\mathrm{n}^{\circ} 2 / 90\right)$. La même loi autorise également la publicité à la radio comme à la télévision. Et elle amorce un processus de réflexion sur le statut des offices de radio et de TV, qui devra être comparable ou identique à celui des établissements de droit public de la RFA.

Or la radio et la TV sont « l'institution de la RDA... qui a survécu le plus longtemps en tant qu'organisation ». Elles étaient un des derniers bastions du régime, et leur 'déSTASIfication' ne s'est souvent faite qu'en apparence ; en réalité, « on y perfectionnait l'agitprop pour mieux défendre ses propres intérêts », se souvient Roland Tichy qui fut conseiller de Rudolf Mühlfenzl et prit une part active à la rénovation de l'audiovisuel de l'ex-RDA (epd medien, $n^{\circ} 78 / 2010$ ). L'art. 36 du Traité d'Unification (signé le 31 août 1990 et entré en vigueur le 3 octobre) avait réuni provisoirement la radio (Rundfunk der DDR) et DFF de l'ex-RDA en une seule structure : la Einrichtung (« l'Institution ») ; ils voyaient ainsi « leur existence assurée jusqu'au 31 décembre 1991 au plus tard, sous la forme d'une personne juridique indépendante de l'Etat et commune aux [nouveaux ; IB] Länder » ; "les
Une démocratisation en plusieurs étapes: institution du Medienkontrollrat...

... réunion de la radio et de la TV au sein d'une Einrichtung à liquider 
Travaux d'Hercule

Deutsche Welle absorbe Radio Berlin International

Quel sort réserver aux autres radios 'internationales'? installations techniques des studios, jusqu'ici propriété de la Deutsche Post, ainsi que les biens immobiliers destinés à la production et à l'administration... lui sont attribués ». Dans l'intervalle, cette Einrichtung est gérée par une sorte de 'syndic de faillite' comprenant deux organes : un plénipotentiaire du gouvernement fédéral pour l'audiovisuel (Rudolf Mühlfenzl, ancien directeur de l'information du Bayerischer Rundfunk, qui prend ses fonctions le 15 septembre 1990) ; il est flanqué par un Comité de la radiodiffusion (Rundfunkbeirat) qui comprend 18 représentants des Länder et des groupes représentatifs de la société. Le $1^{\text {er }}$ janvier 1992, donc, la Einrichtung doit être dissoute par un Contrat d'Etat ad hoc (un Traité conclu entre les Länder, souverains en matière d'audiovisuel), puis transformée en un ou plusieurs établissements de droit public dont il revient aux Länder de fixer les statuts (CIRAC MEDIA, $n^{\circ} 4 / 90$ ). Pour résumer : dès le début de 1990, la RDA avait opté pour le modèle ouest-allemand d'un audiovisuel indépendant de l'Etat et investi d'une mission démocratique, et d'autre part structuré selon les lois du fédéralisme, c'est-à-dire relevant des compétences des législateurs des Länder.

Mais cette tâche est plus complexe qu'il y paraît. Les Länder se constituent rapidement, mais cela implique que chacun, après adoption de sa Constitution, élise son parlement puis son gouvernement (cette phase s'achève le 14 octobre 1990). II leur faut également intégrer 'l'acquis' de l'Allemagne unie : système politique, administratif, législation économique et sociale. Dans ce contexte, "l'Ordre dual de la radiodiffusion » (coexistence du public et du privé, instituée à l'ouest en 1987) s'applique lui aussi automatiquement dans les nouveaux Länder. Reste à chacun d'entre eux de lui donner forme sur son territoire. Autant de travaux d'Hercule à mener de front... S'engagent alors, pour la future configuration de l'audiovisuel public, d'innombrables débats et séminaires réunissant acteurs de la branche, politiques et experts (Kammann, 2010). Mais le climat tourne : l'euphorie des premiers bouleversements retombe après l'Unité, l'immensité du travail de transition, ses manifestations inévitables que sont la montée du chômage et les difficultés rencontrées pour reconstruire l'économie, désorientent l'opinion et suscitent une vague d'Ostalgie (voir dans ce numéro les articles de K. Brenke, E. Spitznagel et T. Petersen). Et les intellectuels, comme une partie de l'opinion projettent alors leur nostalgie sur l'audiovisuel, rêvant de le voir devenir «le dernier bastion de la RDA » (Tichy ; epd medien, $\mathrm{n}^{\circ}$ 78/2010).

Les questions que soulève la réorganisation de l'audiovisuel est-allemand sont elles aussi complexes : quel avenir pour les radios internationales respectives ? quelle configuration donner au service public ? comment mettre en place le secteur privé ?

En ce qui concerne les radios 'internationales', il faut distinguer deux groupes: celles dont la vocation est classique (Deutsche Welle à l'ouest et Radio Berlin International à l'est), et les autres. Les premières finiront par fusionner, Deutsche Welle (Cologne) abosorbant RBI (le choix de Cologne s'explique aussi par la nouvelle répartition des centres de pouvoir au sein de l'Allemagne unie et du choix de Berlin comme capitale). La nouvelle Deutsche Welle lancera un service mondial de TV le $1^{\text {er }}$ avril 1992. La situation des autres est plus délicate à gérer. Du côté ouest, Deutschlandfunk (DLF, Cologne) a largement perdu sa justification (desservir 'l'autre Allemagne') avec l'Unité ; il lui reste sa vocation culturelle et européenne. Du côté est, il y a la station culturelle Radio DDR II ; pourquoi ne pas engager une coopération ? La radio de propagande Deutschlandsender (anciennement Stimme der $D D R$ ) a elle aussi perdu toute légitimité ; il n'en va guère autrement de RIAS (radio de statut américain, mais financée presque exclusivement par la RFA).

II serait dommage de tout simplement liquider ces radios. Et elles attisent les convoitises. L'établissement public de TV commun à tous les Länder, la ZDF (Mayence ; elle édite à l'époque la deuxième chaîne généraliste diffusée dans toute l'Allemagne et participe à la chaîne culturelle 3sat) verrait bien enfin se réaliser son vieux rêve : avoir elle aussi ses stations de radio comme les établisse- 
ments membres de l'ARD, ses concurrents. Comme l'Unité allemande implique la réorganisation de tout l'audiovisuel public, pourquoi ne pas mettre à profit cette occasion pour ajouter un second pilier d'activités au ZDF et intégrer dans ses structures ce Deutschlandfunk qui pourrait à terme, si les législateurs le voulaient bien, avoir à sa disposition un réseau de fréquences lui permettant de desservir aussi l'Allemagne de l'ouest? Car sous la question de l'avenir des structures se profile aussi celle de la réorganisation des plans de fréquences hertziennes terrestres. L'Unité s'est traduite en effet par 'l'addition' des fréquences attribuées à l'ex-RDA et à la RFA ; le départ progressif des forces alliées en libèrera un certain nombre d'autres. Mais une autre question encore se pose : celle de la mission et des statuts. DLF est une radio 'pour l'Europe', et a de ce fait un statut particulier, défini par le Bundestag ; elle relève donc du droit de la Fédération - à l'opposé de l'établissement ZDF qui, lui, repose sur un Contrat d'Etat conclu entre tous les Länder qui lui ont confié la mission de réaliser une chaîne diffusée dans toute l'Allemagne. De surcroît, statutairement, le DLF est membre associé de l'ARD depuis sa création... A l'époque des intenses réflexions autour de l'avenir du DLF, on peine en Allemagne à concevoir qu'une radio puisse faire un programme généraliste 'national' ; ce format est réservé à la TV, les radios étant toutes par définition régionales (KAS, 1991).

Finalement, les ministres-présidents des Länder parviendront à un accord le 25 juin 1992 : il sera créé un organisme doté du statut de collectivité de droit public (Körperschaft des öffentlichen Rechts) chargé de réaliser deux programmes généralistes comprenant tous deux une part importante d'information et d'émissions culturelles. Le choix de ce statut est un compromis : bien qu'il en soit très proche en matière d'autonomie, il diffère de celui d'établissement de droit public (Anstalt des öffentlichen Rechts) qui est celui du ZDF et des membres de l'ARD. Et il permet surtout de concilier toutes les positions tout en préservant les structures existantes en l'état: car une collectivité (à la différence d'un établissement) de droit public est légitimée par l'existence de membres adhérents, en l'occurrence les établissements de l'ARD et le ZDF ; ce sont eux qui la financent. C'est donc l'ensemble du service public de l'audiovisuel allemand qui porte cette nouvelle entité qui prendra pour nom lors de sa constitution en 1994 Deutschlandradio.

Pour concilier les intérêts des Allemands de l'est et de l'ouest, cette entité qui a son siège à Cologne (sa base est le DLF, après avoir transféré ses rédactions en langues étrantères à la Deutsche Welle) dispose de deux centres de production : l'un à Cologne, où est réalisé le programme Deutschlandfunk - il a gardé son nom, mais devient radio 'nationale' allemande, avec une dominante d'information ; l'autre à Berlin, où est réalisée l'antenne baptisée aujourd'hui Deutschlandradio Kultur. Cette radio à dominante culturelle est le fruit de la fusion du premier programme du RIAS (l'autre a été privatisé en 1992) et de DS Kultur, radio qui était née en juin 1990 de la fusion de Radio DDR 2 (culture) et Deutschlandsender (ex-Stimme der DDR). Comme elle mariait deux antagonistes, il était difficile de confier à Deutschlandradio Kultur, du moins dans un premier temps, une mission d'information... Aujourd'hui, les deux antennes de Deutschlandradio couvrent l'Allemagne entière en bande FM - une 'révolution' dans un pays où cette bande était jusque-là utilisée exclusivement par les radios locales et régionales. Et, bien sûr, comme toutes les chaînes et radios allemandes, elles sont diffusées en clair également par les satellites Astra.

Le dossier de la radio fut le dernier à être clos. II impliquait en effet que les structures institutionnelles du service public soient mises en place, que soit revu le mode de répartition interne du produit de la redevance et que, plus généralement, soit fixé le cadre législatif régissant l'ensemble de l'audiovisuel dans l'Allemagne unie. Cette tâche gigantesque fut achevée le $1^{\mathrm{er}}$ janvier 1992, date d'entrée en vigueur du Contrat d'Etat sur la radiodiffusion dans l'Allemagne unifiée (Staatsvertrag über den Rundfunk im vereinten Deutschland), signé par les Länder le 31 août 1991. Ce Contrat étendait aux nouveaux Länder les dispositions en vigueur à l'ouest, tout en déterminant le cadre de la transition entre la liquidation de la

\section{Création de} Deutschlandradio...

... avec 2 antennes 'nationales'

1992 : entrée en vigueur du Contrat d'Etat sur la radiodiffusion dans l'Allemagne unifiée 
A l'automne 1991, création du Mitteldeutscher Rundfunk...
Einrichtung et la mise en service des nouvelles structures publiques, en augmentant la redevance pour faire face à leurs besoins futurs, en modifiant les seuils de concentration pour le secteur privé et en transcrivant en droit national la Directive européenne (publicité). Ce Contrat d'Etat est donc une loi-cadre générale comprenant six lois-cadres particulières (CIRAC MEDIA, $\mathrm{n}^{\circ}$ 8/1991). II se contente au fond d'une part de fixer les règles du jeu (un " ordre " selon le terme allemand) pour les activités du secteur dans toute l'Allemagne et, d'autre part, d'entériner le choix fait par les nouveaux Länder en matière d'organisation de leur audiovisuel public, tout en révisant en conséquence le mode de fonctionnement du financement (système de péréquation) interne au dispositif.

Les nouveaux Länder venaient en effet de décider de se doter d'établissements de radiodiffusion de droit public sur le même modèle que les autres membres de l'ARD. Et à l'automne 1991, leur configuration concrète était elle aussi dessinée. Le premier à voir le jour est le Mitteldeutscher Rundfunk (MDR, Leipzig), créé le 30 mai 1991 par Contrat d'Etat entre les Länder de Saxe, Saxe-Anhalt et Thuringe. Les particularités régionales sont respectées : chaque Land a sa 'maison de la radiodiffusion' pour produire une radio pour le Land, apporter sa contribution aux radios couvrant les 3 Länder signataires et produire des émissions pour les décrochages régionaux de la chaîne commune aux trois Länder.

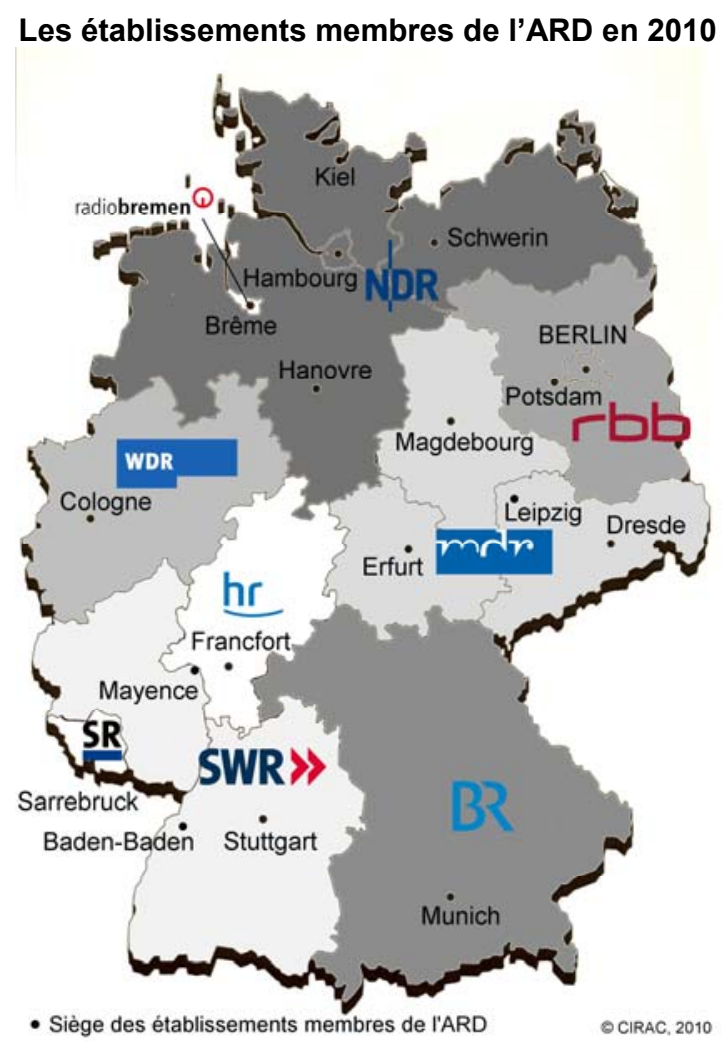

... d'un ORB qui fusionnera en 2003 avec le SFB

Le Land de Meclembourg rejoint le NDR
Le petit Land du Brandebourg (où le produit de la redevance est donc faible) crée par loi le 25 septembre 1991 son propre établissement, contre l'avis de la plupart des experts : Rundfunk Brandenburg ( $R B r$; Potsdam) ; il voulait se démarquer de la trop puissante Berlin. Mais le (trop) petit $R B r$, rebaptisé entre-temps Ostdeutscher Rundfunk Brandenburg (ORB) pour mieux affirmer son identité, finira par fusionner en mai 2003 avec le Sender Freies Berlin de Berlin (ouest) et former l'actuel Rundfunk Berlin Brandenburg (RBB ; Berlin).

Le Land de Mecklembourg avait longtemps hésité, se demandant s'il allait conclure une alliance avec Berlin ou le Brandebourg, ou s'il allait répondre à l'offre du Norddeutscher Rundfunk (NDR) qui couvre alors les anciens Länder entre la Mer du Nord et la Baltique (Basse-Saxe, Hambourg et Schleswig-Holstein) et s'asso- 
cier avec lui. C'est cette option qui l'emportera finalement : le NDR accueille son quatrième partenaire en décembre 1991, après modification du Contrat d'Etat entre les Länder concernés. Pour préserver l'identité du Mecklembourg dans la réalisation de la chaîne généraliste commune destinée au nord de l'Allemagne, le Land a lui aussi, comme les trois autres, sa 'maison de la radiodiffusion' autonome.

Le 31 décembre 1991 à minuit, la Einrichtung cesse donc d'émettre, et l'ARD se présente désormais comme le groupement de travail des établissements de tous les Länder. A l'exception de la récente fusion entre deux établissements du sudouest dans une nouvelle entité (SWR), cette structure n'a plus varié depuis. Le Contrat d'Etat sur la radiodiffusion dans l'Allemagne unifiée est entré en vigueur le $1^{\mathrm{er}}$ janvier 1992. L'Unité de l'audiovisuel public est achevée.

Le modèle ouest-allemand de structures indépendantes de l'Etat, considérées comme des émanations de la collectivité des citoyens (société civile) et contrôlées par cette dernière via ses organes, s'est imposé à l'est. Si l'Intendant dirige l'établissement et en est à la fois 'directeur de la publication' et représentant légal, si le conseil d'administration en contrôle la gestion et donne quitus à l'Intendant, le pluralisme des programmes est garanti par le conseil de surveillance. Ce "Conseil de la radiodiffusion » (Rundfunkrat ; il s'appelle Fernsehrat dans le cas du ZDF) qui contrôle la ligne éditoriale est en effet la concrétisation du contrôle par les citoyens : il se compose de réprésentants de la société civile organisée (fédérations professionnelles, syndicats, Eglises, associations...) et d'un nombre restreint seulement de parlementaires ou de représentants des partis politiques. C'est lui qui élit l'Intendant (et non le Land ou les Länder où l'établissement a son siège). C'était là le mode d'organisation choisi par la jeune RFA pour garantir la fonction de son audiovisuel en démocratie.

Ce mode d'organisation prévaut également pour la régulation du secteur privé de l'audiovisuel. La seule différence est que cet organe de surveillance est externe aux opérateurs privés, et institutionnalisé sous la forme d'établissements de droit public autonomes: les Landesmedienanstalten (littéralement: établissements des médias des Länder). Ces autorités de régulation, qui reposent elles aussi sur la législation d'un Land, ont été instituées entre mai (en Saxe-Anhalt) et juillet 1991 (Mecklembourg). Structures de régulation émanant de la société civile, elles concrétisent elles aussi le principe constitutionnel de l'indépendance de la radiodiffusion vis-à-vis de l'Etat. Financées par la redevance, elles ont une double compétence : elles attribuent les licences d'exploitation aux opérateurs privés de radio et TV sur le territoire du Land; elles contrôlent (a posteriori) le respect du cadre réglementaire (publicité, protection de la jeunesse...) et sanctionnent le cas échéant les contrevenants. Elles sont aujourd'hui au nombre de 15, Berlin et le Brandebourg ayant décidé de se doter d'une réglementation commune pour l'audiovisuel.

\section{Boom du marché des médias et nouvelle donne}

Parallèlement à la reconfiguration organisationnelle et réglementaire du paysage médiatique dans le processus d'unification, le marché est en plein bouleversement. La "valse des alliances » que connaît l'Europe dans la première moitié des années 1990, et où les opérateurs allemands ou implantés sur le marché allemand (le groupe Kirch, les groupes Bertelsmann et RTL) jouent un rôle majeur, est une des manifestations des profonds changements induits à la fois par l'extension du marché que représenta la chute du Mur, et par la multiplication des vecteurs.

Le marché publicitaire explose : au cours de la seule année 1990, les dépenses publicitaires enregistrent une hausse de $6,7 \%$. La presse quotidienne profite peu de ce boom (+1\% seulement), à l'inverse des magazines économiques et d'informations générales $(+13,6 \%)$. Il est vrai que ces derniers avaient lancé notam-
Les nouveaux établissements ont intégré I'ARD

Le contrôle du secteur public est l'affaire de la société

\section{La régulation \\ du secteur privé aussi}


Essor du secteur privé de l'audiovisuel
Pluralisme et diversité du paysage audiovisuel unifié ment une série de dossiers pour expliquer l'économie sociale de marché... La hausse profite essentiellement au média-roi qu'est alors la télévision; ses recettes croissent de 22,5 \%. L'essentiel de cette 'manne' va aux chaînes privées, alors petites encore, mais en pleine progression : cette année-là, pour la première fois, les recettes publicitaires brutes de RTL plus (aujourd'hui $R T L$ ) dépassent celles de l'ARD (961 millions de DM contre 959 millions de DM). La publicité est en effet limitée sur les chaînes publiques: seules la Une et la Deux peuvent en diffuser, et seulement les jours ouvrables, avant 20 heures. Une deuxième petite chaîne privée 'monte' elle aussi : Pro7. Elle voit ses recettes publicitaires nettes augmenter de 214,5 \% (RTL plus : $+134,7$ \%). Ces deux chaînes constituent le cœur de ce qui préfigure les deux grands groupes de TV privés actuels. En peu de temps, la hiérarchie établie est renversée: de 1992 à 1993, ARD et ZDF voient se réduire leurs recettes publicitaires de près de la moitié; elles n'atteignent plus respectivement que 445 et 370 millions de DM. Certes, 1993 est une année de crise économique, ce qui explique en partie cet effondrement, mais plus durables sont les effets de la rapide démultiplication de l'offre de programmes - donc de supports publicitaires après une longue pénurie en comparaison. Aujourd'hui, les opérateurs RTL Group (avec entre autres la chaîne généraliste $R T L$ ) et ProSiebenSat.1 (avec SAT.1) se partagent à peu près à égalité près de $90 \%$ du marché de la publicité TV.

Car c'est immédiatement après l'Unité que se développe réellement le secteur privé. Aux perspectives favorables de financement (essor du marché publicitaire) s'ajoutent en effet deux autres tendances à la fois : l'équipement accéléré des ménages en moyens de réception (câble et satellite) qui élargit potentiellement l'audience et donc le nombre de contacts publicitaires ; et la démultiplication des vecteurs. En 1992, un foyer télévisé ouest-allemand sur trois est 'abonné' au câble (cet abonnement ne concerne que le raccordement au réseau, conçu exclusivement comme un vecteur de distribution); les Allemands de l'est sont $6 \%$ seulement, mais s'équipent de plus en plus volontiers. La Bundespost (ministère des P\&T) qui est alors le seul acteur du câble (infrastructures) d'Allemagne engage un vaste programme de modernisation des installations d'antennes collectives construites du temps de la RDA (elles ont une capacité réduite), tout en poursuivant et développant son 'plan câble 'à l'ouest, puis à l'est.

Si la réorganisation des fréquences hertziennes terrestres permet de dégager progressivement 3 réseaux supplémentaires, c'est surtout le lancement du premier satellite Astra en août 1989 qui accélère l'extension des canaux de diffusion et dans le même temps l'essor de la réception individuelle. Car les satellites précédents, qu'ils soient de télécommunications ou qu'il s'agisse de ce couple franco-allemand de satellites-jumeaux (TDF et TV SAT) destinés à l'audiovisuel (direct broadcasting satellites) mais qui, essentiellement pour des raisons techniques (côté français) et politiques (côté allemand), ne fut jamais réellement mis en service, ne permettaient guère la réception directe et n'avaient qu'un nombre limité de transpondeurs. Presque du jour au lendemain, donc, Astra offrait une douzaine de canaux. En 1989 s'étaient vendues 200000 paraboles (tous satellites confondus), presque exclusivement à l'Ouest. Un an plus tard, il s'en vend 900000 dont le tiers dans l'Est - et presque exclusivement dans les 6 mois suivant l'union monétaire. II s'agit majoritairement de paraboles individuelles permettant de capter Astra.

Aujourd'hui, 20 ans plus tard, la réception de la TV (et même de la radio) par voie hertzienne terrestre est résiduelle en Allemagne: elle ne concerne plus guère qu'environ 1 million de foyers; presque tous les Allemands reçoivent la TV par satellite ou câble, sans parler des liaisons téléphoniques fixes ou mobiles. Et l'offre standard, gratuite, s'élève à une trentaine de chaînes, moitié publiques, moitié privées (voir REA 75/2006). Et si la structure du marché se présente sous la forme d'un oligopole à trois blocs - ARD/ZDF, RTL Group, ProSiebenSat.1 les jalons en ont été posés voici vingt ans : la chute du Mur avait rebattu les cartes, forçant la croissance organique (endogamique) des médias et menant à 
une répartition pluri-médias du marché qui n'a guère varié depuis. La radio est resté le média local ou régional par excellence et est aux mains des éditeurs de quotidiens (majoritairement des PME régionales) ; la TV est toujours le média des géants de la presse (seuls ou en association avec des partenaires issus des médias).

L'UNITÉ DES PAYSAGES MÉDIATIQUES, SI ELLE FUT HOULEUSE, EST ACHEVÉE depuis longtemps. Et son succès est indéniable, tant sous l'angle du marché que de la démocratie. Certes, le groupe des TV publiques d'ARD et ZDF n'a plus qu'une part d'audience cumulée de $40 \%$ environ, mais l'offre d'information n'en est pas moins très diversifiée et surtout de grande qualité en comparaison de nombre d'autres pays européens. Car ce qui domine en RFA, c'est le format de la chaîne généraliste. Même à l'ère du numérique, la TV est et reste avant tout un média d'information, même si, bien entendu, les formats de fiction ou de divertissement sont pléthore. Les 4 grandes chaînes généralistes (la Une de l'ARD, la Deux du ZDF, RTL et SAT.1) diffusent des JT, de même que toutes les 7 chaînes régionales de l'ARD. La presse quotidienne, elle aussi, reste fidèle au modèle du quotidien d'informations générales - même les quotidiens régionaux. Quant à la radio (250 stations), peu segmentée, elle diffuse toujours elle aussi une part importante d'information.

Et dans cette configuration de médias pluralistes, polycentriques, les particularités régionales peuvent s'épanouir, les identités culturelles ou politiques s'affirmer tout en s'insérant dans un ensemble plus vaste, doté de règles communes, qu'est la République fédérale. La devise de la RFA n'est-elle pas, tout comme celle de l'UE au demeurant: " unie dans la diversité »? Depuis le lancement du premier satellite Astra et l'équipement parallèle des ménages en paraboles ou prises câble, les Hambourgeois peuvent regarder la TV des Bavarois, les Berlinois celle des Allemands du nord, les habitants de Cologne celle de la Saxe ou de la Thuringe... C'est peut-être là que réside, bien que personne (?) ne l'ait entrevu à l'époque, l'apport le plus fondamental - au demeurant non intentionnel du satellite Astra : il a créé les conditions techniques permettant un brassage perpétuel des multiples cultures et identités allemandes et ce faisant, soutenu la mission d'intégration dévolue à la télévision.

Si tous les médias contribuent en Allemagne non seulement au bon fonctionnement de la démocratie - tout simplement en assurant le flux permanent d'informations indispensable à un pays et une société où les centres de pouvoirs et de décision politiques, économiques et sociaux sont par nature polycentriques, un flux de surcroît dans l'espace public, sans lequel aucune décision ne pourrait être prise -, ce rôle revient par définition à la télévision. Non seulement la télévision (d'abord de l'ouest, puis de l'est aussi), en assurant l'articulation d'une démocratie renaissante à l'est, a confirmé son rôle constitutionnel de «facteur » dans la formation de l'opinion publique au sens politique, mais elle l'a fait également au sens sociologique. C'est elle surtout qui a assuré et qui assure toujours, malgré toutes les mutations de l'ère numérique, la cohésion de la société allemande. Elle en est à la fois medium et facteur. C'est là le bilan qu'on peut tirer a posteriori du long et difficile processus d'unification des médias. Sans oublier que, parallèlement, les médias ont éminemment contribué à ce que l'Unité devienne une « success story »...

\section{Indications bibliographiques}

\footnotetext{
-ARBEITSgEMEINSChAFT DER LANDESMEDIENANSTALTEN IN DER BUNDESREPUBLIK DEUTSCHLAND (ALM), Jahrbuch2009/2010, Berlin, 2010

- ARBeitSgemeINSChAFT DER LANDESMEDIENANSTALTEN IN DER BUNDESREPUBLIK DEUTSCHLAND (ALM), Fernsehen in Deutschland 2009. Programmbericht, Berlin, 2010

-Bohn R., Hickehier K., Müller E. (eds), Mauer-Show. Das Ende der DDR, die deutsche Einheit und die Medien, Berlin, 1992
} 
-Bourgeois I., «Allemagne : une démocratie construite sur la libre circulation de l'information », Géopolitique, n 108, janvier 2010

-Bourgeois I., " La télévision allemande, une indépendance structurelle », Le Temps des médias, $n^{\circ} 13$, hiver 2009/2010

-Bourgeois I., « Comment la TV ouest-allemande a contribué à la chute du Mur. Un cas unique d'interaction entre médias et opinion », Working paper $n^{\circ} 19 / 1$, novembre 2009 (www.cirac.ucergy.fr) ; version condensée parue in La revue européenne des médias, $n^{\circ} 13$, hiver 2009-2010

-Bourgeols I., «Springer / ProSiebenSat.1 : quel droit de la concurrence ? », Regards sur l'économie allemande, $\mathrm{n}^{\circ} 75,2006$

-Bourgeois I., «Privatrechtliches Fernsehen », in SchWARZKopF D. (ed.), Rundfunkpolitik in Deutschland. Wettbewerb und Öffentlichkeit, vol. 1, Munich, 1999

- Bourgeois I., Radio et télévision publiques en Allemagne. Un modèle à l'épreuve de la nouvelle Europe, Travaux et Documents du CIRAC, 1993 ; et Radios et télévisions privées. Entre la loi et le marché, Travaux et Documents du CIRAC, 1995

- Bourgeols I., "L'audiovisuel en RDA - une longue marche vers la démocratie », Le Monde, supplément Radio télévision, 18/19-02-1990 ; article repris in MÉNUDIER H. (dir.), La RDA 19491990, Asnières, 1990

- Bourgeoıs I., « Allemagne : vers le paysage audiovisuel unifié », Médiapouvoirs, n²0, 1990

- CIRAC MEDIA, Media et communication en RFA, Bulletin d'information trimestriel édité par le CIRAC de janvier 1990 à juin 1993 (14 numéros parus), Archives du CIRAC

- « 'Es gab ein großes Vakuum' », interview de Roland Tichy, epd medien, n 78, 6 octobre 2010

- «'Geistige Mannigfaltigkeit und hohe Qualität», Konzept für ein Hörfunk-Kulturprogramm des Deutschlandsenders (DDR), documenté in epd / Kirch und Rundfunk, n² 43, 2 juin 1990

- Hall P. C. (ed), Ein Bild der deutschen Wirklichkeit. Der Integrationsauftrag des Fernsehens im Prozess der deutschen Einheit, MainzerTage der Fernsehkritik, vol. 24, Mayence, 1992

- Hall P. C. (ed), Revolutionäre Öffentlichkeit. Das Fernsehen und die Demokratisierung im Osten, MainzerTage der Fernsehkritik, vol. 23, Mayence, 1990

- Hans-Bredow-Institut (ed), Das Rundfunkrecht der neuen Bundesländer. Eine kommentierte Dokumentation, Baden-Baden/Hambourg, 1992

- Henle V., «Comment limiter l'expansion de l'audiovisuel public allemand?», Regards sur l'économie allemande, $n^{\circ} 87$, juillet 2008

- HolzWeißıg G., Massenmedien in der DDR, Berlin, 1989

- Kammann U., « Compagnons de voyage. Les médias et l'Unité allemande », Regards sur l'économie allemande, $\mathrm{n}^{\circ}$ 98-99, 2010

-Klump B., Das rote Kloster. Eine deutsche Erziehung, Hambourg, 1978

- KonRad-Adenauer-Stiftung, KAS (ed.), Nationaler Hörfunk - Welches programm für wen? Actes du colloque 'Eicholzer Forum' (Eichholz, 1991), Medienpolitische Werkhefte, Bonn, 1992

- Media Perspektiven. BAsisdaten, Daten zur Mediensituation in Deutschland 2008, Francfort, 2008

- MeYn H., Massenmedien in der Bundesrepublik Deutschland, Berlin, 1994

- Petersen T., «Frères et sœurs dissemblables. Un bilan de l'Unité allemande », Regards sur l'économie allemande, $\mathrm{n}^{\circ}$ 98-99, 2010

-Schabowski G., Das Politbüro. Ende eines Mythos. Eine Befragung. Herausgegeben von SIEREN F., KoEHne L. (eds), Reinbek, 1990

-Schölgens BARTHEL (ed), 25 ahre Urknall : Absichten - Bilanz - Ausblick. Eine medienpolitische Betrachtung, 6. Berliner Medien Diskurs, Sankt Augustin/Berlin, 2009

-Zeitungsjahrbuch DEUtSCHLAND. Meldungen und Meinungen großer deutscher Tageszeitungen. Das Tagebuch der Bundesrepublik und der DDR im letzten Jahr ihrer Existenz, Munich, 1990 